\title{
Fear of Crime, Consumption, and Culture: An Abstract
}

\author{
Enrique P. Becerra and Maria Cecilia Henriquez-Daza
}

\begin{abstract}
Fear of crime is increasing worldwide despite falling crime rates, but little is known about its effects on consumers and consumption. Fear of crime increases stress, reduces health, creates no-go areas, changes consumer patterns of behavior, affects asset values, and reduces conspicuous consumption, among other effects (Becerra and Henriquez-Daza 2011; Mesch 2000; Moore and Shepherd 2006; Pope 2008; Riger and Gordon 1981; Visser et al. 2013). Fear of crime is cognitive and emotional assessment of perceived crime threats; it is a signal informing us of dangerous situations. Crime threats are assessed through perceptions of victimization and fear. Victimization risk relates to probability of becoming a victim of crime, and fear relates to emotional and/or physiological response to crime threats (Warr 2000). Drawing from literature across various disciplines, we postulate a framework to understand phenomenon, and test it using data from two countries to determine if cultural orientation affects influence of fear of crime on behavior. Findings indicate consumers' behavior is negatively affected by fear of crime, but this effect varies across cultural orientations.
\end{abstract}

References Available Upon Request

\author{
E. P. Becerra $(\square)$ \\ Texas State University, San Marcos, TX, USA \\ e-mail: eb25@txtsate.edu \\ M. C. Henriquez-Daza \\ Pontificia Universidad Javeriana, Cali, Colombia \\ e-mail: mchenriquez@javerianacali.edu.co
}

